February 1994

INFNCA-TH-94-4

\title{
DUALITIES OF LORENTZIAN AND EUCLIDEAN BLACK HOLES IN TWO-DIMENSIONAL STRING-GENERATED MODELS
}

\author{
M. Cadoni \\ S. Mignemi \\ Dipartimento di Scienze Fisiche, Università di Cagliari \\ and Istituto Nazionale di Fisica Nucleare, Sezione di Cagliari \\ Via Ada Negri 18, I-09127 Cagliari, Italy
}

\begin{abstract}
We discuss the properties of lorentzian and euclidean black hole solutions of a generalized 2-dim dilaton-gravity action containing a modulus field, which arises from the compactification of heterotic string models. The duality symmetries of these solutions are also investigated.
\end{abstract}


Two-dimensional dilaton-gravity theories have been studied for several reasons. First of all, they are a theoretical laboratory for investigating some features of gravity which are out of our reach in four dimensions [1]. Moreover, they have great relevance in the study of 2-dim conformal field theories and consequently of exact propagation of strings in fixed backgrounds [2]. Finally, they arise naturally as effective 2-dimensional models of the low-energy 4-dimensional string theory and are especially helpful in the study of the scattering of radiation from the corresponding black hole solutions [3].

Studying an effective 4-dim string model, where non trivial couplings of modulus fields arising from one-loop string threshold effects were taken into account [4], we were lead to consider a 2-dim modulus-dilaton-gravity model [5] whose essential feature is a 1-parameter action, which contains as special cases most of the 2-dim models proposed till now. Starting from a quite different approach, a model leading to equivalent solutions was obtained in ref. [6]. In this letter, we wish to study some general features of the lorentzian and euclidean black hole solutions of these theories and discuss the duality transformations which connect some of them. We also comment about the significance of such duality transformations from the point of view of string theories.

Let us consider the 2-dim action:

$$
S=\frac{1}{2 \pi} \int \sqrt{g} d^{2} x e^{-2 \phi}\left[R+4(\nabla \phi)^{2}-\frac{2}{3}(\nabla \psi)^{2}+\lambda_{1}^{2}-\lambda_{2}^{2} e^{2\left(\phi-\frac{q}{3} \psi\right)}\right],
$$

where $\phi$ is the dilaton field and $\psi$ a modulus field, $q$ being a coupling constant. This action was derived as an effective 2-dim action for heterotic string models in the extremal black hole limit, when modulus fields are taken into account [5].

It was shown in [5] that exact solutions can be obtained for any value of $q$, provided that $e^{\psi}=$ const $\times e^{3 \phi / q}$. In this case the action (1) simplifies to:

$$
S=\frac{1}{2 \pi} \int \sqrt{g} d^{2} x e^{-2 \phi}\left[R+\frac{8 k}{k-1}(\nabla \phi)^{2}+\Lambda\right]
$$

where $k=\frac{3-2 q^{2}}{3+2 q^{2}},-1 \leq k \leq 1$ and $\Lambda=\lambda_{2}^{2}$.

In this form, the action admits several interesting special cases for given values of $k$ [6]. The limit $k=-1$ (i.e. $q \rightarrow \infty$ ) is the string theoretic case $[2,3]$. For $k=0$, one obtains the Jackiw-Teitelboim theory [1], while $k=1$ is the 2-dim equivalent of General Relativity [8, 9].

Black hole solutions of (2), or equivalently of (1), subject to the condition $e^{\psi}=$ const $\times$ $e^{3 \phi / q}$, can be easily found, for any value of $k$, in the unitary gauge $d s^{2}=-e^{2 \rho(\sigma)} d t^{2}+d \sigma^{2}$ $[5,6]$. They are given by:

$$
\begin{gathered}
d s^{2}=-\sinh ^{2}(\kappa \sigma) \cosh ^{2 k}(\kappa \sigma) d t^{2}+d \sigma^{2}, \\
e^{2\left(\phi-\phi_{0}\right)}=\cosh ^{k-1}(\kappa \sigma),
\end{gathered}
$$

where $\kappa=\sqrt{\frac{|\Lambda|}{2(1-k)}}$. Of course, in the limit $k \rightarrow-1$, the solution reduces to that found in $[2,10]$. 
Another class of solutions of (2) is given by anti-de Sitter spacetime with linear dilaton:

$$
\begin{gathered}
d s^{2}=-e^{2(k+1) \kappa \sigma} d t^{2}+d \sigma^{2}, \\
e^{2\left(\phi-\phi_{0}\right)}=e^{(k-1) \kappa \sigma},
\end{gathered}
$$

(flat space for $k=-1$ ). This is the quantum ground state of the theory [5].

Also of interest is the case in which the effective cosmological constant $\Lambda$ is negative, even if this is not compatible with string theory. In this case, the solutions are periodic in $\sigma$ and are given by:

$$
\begin{gathered}
d s^{2}=-\sin ^{2}(\kappa \sigma) \cos ^{2 k}(\kappa \sigma) d t^{2}+d \sigma^{2}, \\
e^{2\left(\phi-\phi_{0}\right)}=\cos ^{k-1}(\kappa \sigma) .
\end{gathered}
$$

As special cases, for $k=0,1$, one gets 2-dim de Sitter spacetime .

It is interesting to notice the existence of a duality symmetry in the action (1), which generalizes that found in [7] in the $k=-1$ case.

In fact, substituting for the Ricci scalar its value $R=-2 e^{2 \rho}\left(\rho^{\prime \prime}+\rho^{2}\right)$ in the unitary gauge, the action can be written:

$$
S=\int d^{2} x e^{-2 \phi+\rho}\left[-2\left(\rho^{\prime \prime}+\rho^{\prime 2}\right)+4 \phi^{\prime 2}-4 \frac{1+k}{1-k} \tilde{\psi}^{\prime 2}+\lambda_{1}^{2}-\lambda_{2}^{2} e^{2(\phi-\tilde{\psi})}\right],
$$

where $\tilde{\psi}=q \psi / 3$. One can then readily check that the transformation:

$$
\begin{aligned}
\rho & \rightarrow k \rho-2(k+1) \tilde{\psi}, \\
\phi & \rightarrow \frac{k-1}{2} \rho+\phi-(k+1) \tilde{\psi}, \\
\tilde{\psi} & \rightarrow-k \tilde{\psi}+\frac{k-1}{2} \rho
\end{aligned}
$$

leaves the action invariant, modulo a total derivative. ${ }^{\dagger}$

The effect of the transformations (7) on the black hole solutions (3) is to exchange the hyperbolic sine and cosine everywhere:

$$
\begin{gathered}
d s^{2}=-\cosh ^{2}(\kappa \sigma) \sinh ^{2 k}(\kappa \sigma) d t^{2}+d \sigma^{2}, \\
e^{2\left(\phi-\phi_{0}\right)}=\sinh ^{k-1}(\kappa \sigma) .
\end{gathered}
$$

The solutions (4), instead, are self-dual, i.e. invariant under the transformation (7). Analogously, the compact solutions (5) are not affected by the duality transformations, except for a shift of the coordinate $\sigma$, changing the sine into cosine.

$\dagger$ We remark that the reduced action (2) is of course invariant under the transformations: $\rho \rightarrow k \rho-2(k+1) \phi ; \phi \rightarrow \frac{k-1}{2} \rho-k \phi$. 
The properties of the Lorentzian black holes have been discussed in extent in [5] and [6]. We give here a short review, add some remarks and discuss the effect of the duality transformations on the solutions.

In order to study the black hole solutions, it is useful to write the metrics in Schwarzschild coordinates. In such coordinates it is in fact possible to continue the metrics besides the horizon at $\sigma=0$ and to get a more immediate insight of their physical properties.

The new coordinates are defined so that the metric takes the form $d s^{2}=-A(r) d t^{2}+$ $A^{-1}(r) d r^{2}$. The general solutions in this gauge are:

$$
\begin{gathered}
d s^{2}=\left(b r^{2}-a r^{\frac{2 k}{1+k}}\right) d t^{2}+\left(b r^{2}-a r^{\frac{2 k}{1+k}}\right)^{-1} d r^{2}, \\
e^{2 \phi}=(\sqrt{b} r)^{\frac{k-1}{k+1}},
\end{gathered}
$$

where $b=\frac{(1+k)^{2} \Lambda}{2(1-k)}$ and $a$ is an integration constant.

The curvature scalar is given by:

$$
R=-2\left(b+\frac{k(1-k)}{(1+k)^{2}} a r^{\frac{-2}{1+k}}\right) .
$$

For generic $k$ (i.e. $k \neq 0, \pm 1$ ), there are four different cases:

a) $\Lambda>0, a>0$ :

These are the solutions (3), obtained from the previous expression by the change of variables $r=(a / b)^{\frac{1+k}{2}} \cosh ^{1+k}(\kappa \sigma)$. They correspond to asymptotically anti-de Sitter black holes with horizon at $r_{0}=(a / b)^{\frac{1+k}{2}}$ and singularity at $r=0$.

b) $\Lambda>0, a<0$ :

These are the duals of the previous solutions. They can be obtained from (9) with the change of variables $r=(-a / b)^{\frac{1+k}{2}} \sinh ^{1+k}(\kappa \sigma)$. A naked singularity is present at finite distance $(r=0)$.

c) $\Lambda>0, a=0$ :

This case corresponds to anti-de Sitter spacetime (4). It is an everywhere regular spacetime.

d) $\Lambda<0, a>0$ :

For negative $\Lambda, a$ must be positive in order to have the correct signature for the metric. These spacetimes correspond to de-Sitter background with a naked singularity at the origin and a cosmological horizon at $r_{0}=\left(\frac{a}{b}\right)^{\frac{1+k}{2}}$. They can be interpreted as the interior of the black holes (a).

The black holes solutions can be maximally extended by introducing Kruskal coordinates. In case (a) they can be obtained by defining a new coordinate (for simplicity we set $|a|=|b|=1)$ :

$$
r^{*}=\frac{1}{k+1} \int \frac{d r}{r^{2}-r^{\frac{2 k}{1+k}}}=\frac{r^{\frac{1-k}{1+k}}}{k-1} \mathrm{~F}\left(\frac{1-k}{2}, 1, \frac{3-k}{2}, r^{\frac{2}{1+k}}\right),
$$


where $\mathrm{F}$ is the hypergeometric function, and

$$
u=e^{r^{*}+t}, \quad v=e^{r^{*}-t}
$$

giving rise to

$$
d s^{2}=(k+1)^{2}\left(r^{2}-r^{\frac{2 k}{1+k}}\right) \exp \left[-\frac{2 r^{\frac{1-k}{1+k}}}{k-1} \mathrm{~F}\left(\frac{1-k}{2}, 1, \frac{3-k}{2}, r^{\frac{2}{1+k}}\right)\right] d u d v
$$

where $r$ is defined implicitly in terms of $u v$ by (11) and (12).

In these coordinates, the metric is clearly regular at the horizon $u v=0$, and in general singular at $u v=1$, i.e. $r=0$, excepted the special cases $k=0,1$. Spatial infinity is represented by the curve $u v=-1$. The Kruskal diagram for a generic solution is shown in figure (1). It is quite similar to that of a Schwarzschild -anti-de Sitter black hole. The region I corresponds to the external spacetime, whose spatial infinity is given by the curve $u v=-1$. Region II corresponds to the black hole interior. An observer in region II will fall in a finite proper time into the singularity at $u v=1$. Regions III and IV have the usual white hole interpretation.

In the $k=-1$ limit, as shown by Giveon [7], region $\mathrm{V}$ corresponds to the dual black hole, with the naked singularity at $u v=1$. In fact, in that limit, the Kruskal coordinates have the same analytical form in both cases, but with the regions I and V interchanged. This is a consequence of the dual symmetry [7]. We believe that something analogous happens also in the more general cases. However, due to the intricate analytical form of the metric (11) we were not able to check this fact explicitly, except for $k=0$

One can classify the solutions according to the value of their masses and of their thermodynamical parameters. The mass can be easily obtained in cases (a) and (b) by means of the ADM procedure. In the Schwarzschild gauge the result is [5]:

$$
M=\frac{(1-k) b^{\frac{1-k}{2(1+k)}}}{2(1+k)} a \text {. }
$$

The sign of the mass coincides therefore with that of the constant $a$ : the dual solutions have negative mass. This is analogous to 4-dim GR, where naked singularities correspond to negative masses.

The temperature is given as usual by the inverse periodicity of the regular euclidean solution and can be obtained by using the standard formula for the Schwarzschild coordinates [11]:

$$
T=\left.\frac{1}{4 \pi} \frac{d}{d r} g_{00}\right|_{r=r_{0}}=\frac{a^{\frac{1+k}{2}} b^{\frac{1-k}{2}}}{2 \pi(k+1)}=\frac{1}{2 \pi}\left(\frac{2 M}{1-k}\right)^{\frac{1+k}{2}}\left(\frac{\sqrt{b}}{1+k}\right)^{\frac{1-k}{2}}
$$

This is the temperature associated with the horizon in case (a). It is apparent that it is a monotonically increasing function of the mass (at variance with 4-dim General Relativity) 
for any value of $k$, except the limit case $k=-1$, when it is independent of $M, T=\frac{\sqrt{b}}{4 \pi}$. In the other limit case, $k=1$, one has $T=\frac{M}{4 \pi}$. In every case except $k=-1, M=0$ is therefore the endpoint of the semiclassical Hawking evaporation process.

The entropy can be most easily obtained by integrating the thermodynamical relation $d S=T^{-1} d M$, resulting in:

$$
S=2 \pi\left(\frac{1+k}{1-k} \frac{2 M}{\sqrt{b}}\right)^{\frac{1-k}{2}} .
$$

Again, we have a positive power function of $\mathrm{M}$, with the exception of $k=1$, when the entropy is given by $S=4 \pi \ln M$ [8]. A remarkable relation, valid whenever $k \neq 1$ is $S T=\frac{2 M}{1-k}$.

It should be noticed that the formulae above are formally valid also for the cosmological horizon in case $(\mathrm{d})$.

It is also interesting to study the Newtonian limit of the black hole solutions (a). If one neglects matter couplings to the dilaton, this is given as in GR by the linearization of $g_{00}$. Besides the long-range force due to the anti-de Sitter asymptotic behaviour, one can have quite different short-range behaviour depending on the sign of $k$ : the short-range potential is in fact given by $-a r^{\frac{2 k}{1+k}}$, it can therefore either diverge or go to zero at the origin. Moreover, the short-range force is repulsive if $k>0$ (see figure 2). This fact causes a very different behaviour in the quantum evaporation process [5].

We discuss now in some detail the case $k=0$. This is the simplest non-trivial example, but nevertheless possesses very interesting solutions, among which a non-singular black hole [12] and can clarify some features of the more general case. Its physical interest derives from its equivalence to the Jackiw-Teitelboim theory [1]. The other special cases, $k= \pm 1$, are discussed in detail in $[2,10]$ and $[8]$.

As is easily seen, the solutions of the theory have constant curvature $-\Lambda$ and the dilaton, in the Schwarzschild gauge, is proportional to $r^{-1}$. Contrary to the general case, there are no curvature singularities. In two dimensions there exist several different manifolds with negative constant curvature: the solutions can indeed be divided in four classes: ${ }^{\dagger}$

a) $\quad \Lambda>0, \quad a>0 ; \quad d s^{2}=-\left(r^{2}-1\right) d t^{2}+\left(r^{2}-1\right)^{-1} d r^{2}=-\sinh ^{2} \sigma d t^{2}+d \sigma^{2}$.

These solutions are non-singular black holes with a horizon at $r=1$ and anti-de Sitter asymptotic behaviour. They have been studied in ref [12]. The change of coordinates

$$
u=\sqrt{\frac{r-1}{r+1}} e^{t}, \quad v=-\sqrt{\frac{r-1}{r+1}} e^{-t},
$$

takes the metric into the Kruskal form:

$$
d s^{2}=-4 \frac{d u d v}{(1+u v)^{2}}
$$

with dilaton field:

$$
e^{2\left(\phi-\phi_{0}\right)}=\frac{1+u v}{1-u v}
$$

$\dagger$ For simplicity we put $|a|=|b|=1$ in the following discussion. 
The Kruskal diagram is depicted in fig. 3. The Schwarzschild coordinates cover the region I of the diagram (the region IV reversing the signs of both $u$ and $v$ ). The curves $u v=0$ are of course the horizon $r=1$, while the hyperbola $u v=-1$ represents spatial infinity.

The metric can be realized in 3-dim Minkowski space of signature $\left(--_{-}+\right)$, by considering a 2-sheet hyperboloid of equation $x^{2}+y^{2}-z^{2}=-1$ with the choice of coordinates:

$$
x=\sinh \sigma \sin t, \quad y=\sinh \sigma \cos t, \quad z=\cosh \sigma .
$$

The vertex of the hyperboloid corresponds to the horizon. This geometric construction displays intuitively the regularity of the metric.

b) $\quad \Lambda>0, a<0 ; \quad d s^{2}=-\left(r^{2}+1\right) d t^{2}+\left(r^{2}+1\right)^{-1} d r^{2}=-\cosh ^{2} \sigma d t^{2}+d \sigma^{2}$.

This metric is dual to (a) and can be readily recognized to be the usual 2-dim anti-de Sitter spacetime. It is an everywhere regular solution and can be realized analogously to case (a) in 3-dim Minkowski space, but this time by a 1-sheet hyperboloid of equation $x^{2}+y^{2}-z^{2}=1$ parametrized as:

$$
x=\cosh \sigma \sin t, \quad y=\cosh \sigma \cos t, \quad z=\sinh \sigma .
$$

The Kruskal coordinates can be defined as:

$$
u=\tan \left[\frac{1}{2}(t+\arctan r)\right], \quad v=\tan \left[\frac{1}{2}(t-\arctan r)\right],
$$

so that the metric takes the same form as (17). The original coordinates, however, cover in this case the region $\mathrm{V}$ of figure 3 (the region VI reversing the signs of both $u$ and $v$ ), the hyperbola $u v=-1$ representing spatial infinity, and the dilaton field is given by:

$$
e^{2\left(\phi-\phi_{0}\right)}=\frac{1+u v}{u-v}
$$

c) $\quad \Lambda>0, \quad a=0 ; \quad d s^{2}=-r^{2} d t^{2}+r^{-2} d r^{2}=-e^{2 \sigma} d t^{2}+d \sigma^{2}$.

We finally consider the case where $a=0$. This is like (b) anti-de Sitter spacetime, but with a different parametrization. Now, the 1-sheet hyperboloid of (b) is in fact parametrized by the coordinates

$$
x=e^{\sigma} t, \quad y=\cosh \sigma-\frac{1}{2} e^{\sigma} t^{2}, \quad z=\sinh \sigma+\frac{1}{2} e^{\sigma} t^{2},
$$

which however only cover half of it. One can define Kruskal coordinates,

$$
u=\frac{1}{r}+t, \quad v=\left(\frac{1}{r}-t\right)^{-1},
$$

such that the metric has again the form (17). In this case $u$ and $v$ can take any real value and the metric is in fact self-dual.

d) $\quad \Lambda<0, \quad a<0 ; \quad d s^{2}=-\left(1-r^{2}\right) d t^{2}+\left(1-r^{2}\right)^{-1} d r^{2}=-\sin ^{2} \sigma d t^{2}+d \sigma^{2}$. 
Case (d) is de Sitter spacetime. As is well known it can be represented by a 1-sheet hyperboloid of equation $x^{2}+y^{2}-z^{2}=1$, like anti-de Sitter , but with the signature of the embedding space changed to $(+,+,-)$. The metric is regular, but a cosmological horizon is present at $r=1$. This spacetime can be interpreted as the interior of the black hole (a). In Kruskal coordinates,

$$
u=\sqrt{\frac{1-r}{1+r}} e^{t}, \quad v=\sqrt{\frac{1-r}{1+r}} e^{-t},
$$

the form of the metric and of the dilaton is in fact again (17), and the range of the coordinates covers the region II of figure 3 (the region III reversing the signs of both $u$ and $v)$.

We pass now to consider the euclidean black hole solutions of our theory. These are relevant in the sigma-model formulation of 2-dim conformal field theories and have nice geometric properties. They can be simply obtained by analytical continuation of the lorentzian solutions; for $\Lambda>0$ :

$$
\begin{gathered}
d s^{2}=\sinh ^{2}(\kappa \sigma) \cosh ^{2 k}(\kappa \sigma) d \tau^{2}+d \sigma^{2}, \\
e^{2\left(\phi-\phi_{0}\right)}=\cosh ^{k-1}(\kappa \sigma),
\end{gathered}
$$

or, for the dual case,

$$
\begin{aligned}
d s^{2}= & \cosh ^{2}(\kappa \sigma) \sinh ^{2 k}(\kappa \sigma) d \tau^{2}+d \sigma^{2}, \\
& e^{2\left(\phi-\phi_{0}\right)}=\sinh ^{k-1}(\kappa \sigma),
\end{aligned}
$$

where $\tau$ is the euclidean time and $\kappa$ is defined as in eq. (3). All the solutions but $k=-1$ tend asymptotically to euclidean anti-de Sitter space, which is also a solution of the field equations:

$$
\begin{gathered}
d s^{2}=e^{2(k+1) \kappa \sigma} d \tau^{2}+d \sigma^{2}, \\
e^{2\left(\phi-\phi_{0}\right)}=e^{2(k-1) \kappa \sigma} .
\end{gathered}
$$

All the solutions (19) are regular for $0<\sigma<\infty$ and $0 \leq \tau \leq \beta$, where $\beta=2 \pi \sqrt{\frac{2(1-k)}{\Lambda}}$ is the inverse temperature in this gauge, and are therefore periodic in $\tau$. The dual solutions (20) are instead singular at $\sigma=0$. The corresponding geometries are depicted in figure 4 .

In the case $k=-1$ the solutions have the well known "cigar" and "trumpet" shape $[2,7]$ and are asymptotically a flat cylinder. In the other cases, instead, the solutions (19) have the shape of an infinite hood, which asymptotes a cone at infinity. The dual solutions (20) are similar, but with a singularity at finite distance at $\sigma=0$, except the cases $k=0,1$, when they are regular everywhere and can be represented as hyperboloids in 3-dim Minkowski space. The volume and the action of these solutions are of course infinite, so they cannot be interpreted as instantons in the usual sense.

In the case $\Lambda<0$ the solutions are instead compact. They are periodic both in $\sigma$ and $\tau$ and are given by:

$$
d s^{2}=\sin ^{2}(\kappa \sigma) \cos ^{2 k}(\kappa \sigma) d \tau^{2}+d \sigma^{2}
$$




$$
e^{2\left(\phi-\phi_{0}\right)}=\cos ^{k-1}(\kappa \sigma) .
$$

When $k=0,1$ they are 2 -spheres. In the other cases they are surfaces of revolution, singular at $\sigma=0$. They have finite volume $\frac{8 \pi \Lambda}{1+k}$, but infinite action. Only for $k=-1$ the volume diverges, but the action becomes finite.

To conclude, we add some remarks about the meaning of the duality symmetry of the action (1) at a string theoretic level. The issue one wants to clarify is the relationship between the transformations (7) and the target space duality transformations of the effective action of the string. A related, and even more fundamental question concerns the possibility that the background defined by (3) has a description in terms of a 2-dim conformal field theory on the worldsheet. In ref. [5] the action was derived as an effective 2-dim gravity action stemming from a 4-dim effective string action in which string one-loop threshold effects were taken into account.

It seems impossible to relate directly the symmetries of the 2-dim effective theory to those of the original model from which it was derived. However, some insight into the subject can be achieved by considering the 2-dim theory as a toy model for 2-dim dilatonic gravity coupled to a modulus field.

We shall assume that the field $\psi$ appearing in the action (1) parametrizes the radius $\mathcal{R}$ of some compactified manifold $\left(\mathcal{R}=e^{-\psi}\right)$. In the large radius limit, which can be achieved by letting $q \rightarrow \infty$, the potential term for the field $\psi$ disappears from the action:

$$
S=\frac{1}{2 \pi} \int \sqrt{g} d^{2} x e^{-2 \phi}\left[R+4(\nabla \phi)^{2}-\frac{2}{3}(\nabla \psi)^{2}+\lambda_{1}^{2}\right] .
$$

The ensuing equations of motion force the field $\psi$ to be constant, so that the general solutions, in the unitary gauge, are:

$$
e^{2 \rho}=\tanh ^{2}(\kappa \sigma), \quad e^{2\left(\phi-\phi_{0}\right)}=\cosh ^{-2}(\kappa \sigma), \quad e^{-2 \psi}=\text { const } .
$$

The spacetime described by these solutions can be thought as the $D=3$ conformal background of ref. [13], corresponding to the uncharged black string spacetime $S L(2, R) / U(1) \times$ $U(1)$, obtained by attaching a circle of constant radius to every point of the $D=2$ black hole spacetime of the $S L(2, R) / U(1)$ gauged WZW model.

The action (23) in the unitary gauge possesses two independent sets of duality symmetries:

$$
\begin{gathered}
\psi \rightarrow-\psi, \\
\rho \rightarrow-\rho, \quad \phi \rightarrow-\rho+\phi .
\end{gathered}
$$

The transformations (25.a) corresponds to the well-known $\mathcal{R} \rightarrow 1 / \mathcal{R}$ circle duality, whereas the transformations (25.b) is the target space duality of ref. [7].

The former dualities are symmetries of the action (1) only in the large radius limit. The exact duality symmetries of the action (1) are represented by eq. (7), from which we recover in the limit $q \rightarrow \infty$ the form (25).

In conclusion, the introduction of the term $\lambda_{2}^{2} e^{-2 q \psi / 3}$ in the action (23) not only modifies radically the form of the solutions, allowing for non-constant values of the field 
$\psi$, but also breaks the duality symmetries (25). The general action (1) is, however, still invariant under the duality transformation (7). It remains open the question whether the background defined by (3) has an interpretation as a 2-dim conformal field theory. Work in progress indicates that this is the case at least for $k=0$. In fact in this case the solution describes the group invariant metric of $\mathrm{SL}(2, \mathrm{R})$ and corresponds to the extremal limit of the charged black string of ref. [13].

\section{References}

[1] C. Teitelboim, in Quantum Theory of gravity, S.M. Christensen, ed. (Adam Hilger, Bristol, 1984); R. Jackiw, ibidem;

[2] E. Witten, Phys. Rev. D44, 314 (1991);

[3] C.G. Callan, S.B. Giddings, J.A. Harvey and A. Strominger, Phys. Rev. D45, 1005 (1992);

[4] M. Cadoni and S. Mignemi, Phys. Rev. D48, 5536 (1993);

[5] M. Cadoni and S. Mignemi, preprint INFN-CA-20-93, hep-th 9312171;

[6] J.P.L. Lemos and P.M. Sá, Preprint DF/IST-9.93, to appear on Phys. Rev. D;

[7] A. Giveon, Mod. Phys. Lett. A6, 2843 (1991);

[8] R.B. Mann, A. Shiekh and I. Tarasov, Nucl. Phys. B341, 134 (1990);

R.B. Mann, Gen. Rel. Grav. 24, 433 (1992);

R.B. Mann, T.G. Steele, Class. Quantum Grav. 9, 475 (1992);

[9] J.P.L. Lemos and P.M. Sá, Preprint DF/IST-17.93, to appear in Class. Quantum Grav.

[10] G. Mandal, A.M. Sengupta and S.R. Wadia, Mod. Phys. Lett. A6, 1685 (1991);

[11] J.W. York, Phys. Rev. D31, 775 (1985);

[12] J.P.L. Lemos and P.M. Sá, Preprint DF/IST-8.93;

[13] S.H. Horne and G.T. Horowitz, Nucl. Phys. B368, 444 (1992). 
This figure "fig1-1.png" is available in "png" format from: http://arxiv.org/ps/hep-th/9403113v1 
This figure "fig1-2.png" is available in "png" format from: http://arxiv.org/ps/hep-th/9403113v1 
This figure "fig1-3.png" is available in "png" format from: http://arxiv.org/ps/hep-th/9403113v1 
This figure "fig1-4.png" is available in "png" format from: http://arxiv.org/ps/hep-th/9403113v1 